\title{
DETERMINAÇÃO DA COMPOSIÇÃO QUÍMICA E POTENCIAL ANTIOXIDANTE DO AZEITE DE OLIVA PRODUZIDO NA REGIÃO OESTE DE SANTA CATARINA
}

\author{
T. T. S. HUK ${ }^{1}$, J. SCAPINELLO ${ }^{1}$, D. M. CROCE ${ }^{2}$, F. KUHN ${ }^{1}$, A. BOHN ${ }^{1}$, J. DAL \\ MAGRO $^{1}$ \\ ${ }^{1}$ Universidade Comunitária da Região de Chapecó, Área de Ciências Exatas e \\ Ambientais \\ ${ }^{2}$ Centro de pesquisa para agricultura familiar - Empresa de Pesquisa Agropecuária e \\ Extensão Rural de Santa Catarina - EPAGRI \\ E-mail para contato: jacir@uncohapeco.edu.br
}

\begin{abstract}
RESUMO - Atualmente o Brasil depende de importações de azeite de oliva para atender seu mercado, e a partir do cultivo de oliveiras em solo brasileiro visa-se redução de custos no processo. Assim surge a necessidade de avaliar a composição química do azeite proveniente dessas plantações. Para azeites das espécies Koroneiki, Arbequina e Arbosana, cultivadas no oeste de Santa Catarina, determinou-se o teor de acidez, índice de peróxido, índice de iodo (Wijs), extinção específica por absorção em $270 \mathrm{~nm}$ e potencial antioxidante (DPPH). Para a acidez obteve-se resultados na faixa de 0,261 a $0,719 \mathrm{~g} / 100 \mathrm{~g}$. Índice de peróxido entre 10,46 a $17,36 \mathrm{meq} / \mathrm{kg}$. Valores entre 59,4 e $105,3 \mathrm{mg} / \mathrm{kg}$ para compostos fenólicos, e índice de iodo entre 76,7 e 93,1. O potencial antioxidante foi de 1,34 a $18,1 \%$. O índice de extinção para todas as amostras ficou abaixo de 0,25 . Todos os resultados obtidos estão de acordo com valores previstos na legislação vigente, indicando excelente qualidade dos azeites produzidos.
\end{abstract}

\section{INTRODUÇÃO}

Entre os séculos VII e III a.C. o azeite de oliva começou a ser estudado pelos filósofos, médicos e historiadores da época pelas suas propriedades benéficas ao ser humano (Percussi, 2006). Nos últimos anos, inúmeros estudos têm apresentado evidências de que nutrientes e substâncias não-nutrientes contidas em diferentes alimentos, como o azeite de oliva, podem inferir de modo positivo na prevenção de enfermidades. Estudos experimentais e epidemiológicos sugerem que o azeite de oliva extra virgem, com alto teor de compostos fenólicos, exerce papel protetor contra doenças crônicas degenerativas, inclusive a aterosclerose, pelo conteúdo de gorduras monoinsaturadas (ácido oleico) e poder antioxidante (Pimentel et al., 2007).

Desde 2003 o maior produtor mundial de azeite é a Espanha com 60\% da produção mundial, seguido por Itália, Grécia, Síria e Turquia. Países como Estados Unidos, Argentina e 
Chile são considerados produtores menos significativos, e há ainda países que tentam adaptar a oliveira ao seu solo e clima, como o Brasil, mas possuem produção ainda muito pequena (Aparicio, 2003).

Planta característica de clima temperado, a oliveira necessita de baixas temperaturas no período que antecede a floração, altitudes que variam entre 200 e 1300 metros e regime de chuvas superior a $800 \mathrm{~mm}$ para que se obtenham resultados satisfatórios na produção de azeites. $\mathrm{O}$ pH do solo, que deve ser superior a 5,5, também interfere na qualidade da produção (Coutinho et al., 2009). Distingue-se na espécie Olea europaea L. diversas variedades cujas diferenças podem ser causadas pelo solo e região de cultivo, pelo clima, entre outros fatores, aumentando o teor de azeite, o volume do fruto, o tamanho do caroço e o teor e consistência da polpa (Peixoto et al., 1973).

Como não existe uma tradição olivícola no Brasil, o país depende de importações para abastecer o mercado interno, e os impostos incidentes sobre a importação encarecem o produto. A produção de oliveiras em solo brasileiro, apesar de algumas regiões apresentarem clima bastante favorável, ainda é baixa. Nos últimos 30 anos, algumas empresas de pesquisa agropecuária do país estão apostando no cultivo da planta, visando a produção de azeite nacional (Peixoto, 1998). Surge então, a necessidade de avaliar a composição química do azeite produzido em solo brasileiro, a fim de garantir qualidade equivalente ou, até mesmo, superior aos azeites importados de outros países. Segundo o Codex Alimentarius (2003), o padrão de qualidade de azeites deve ser avaliado através da determinação de parâmetros como teor de acidez, índice de peróxidos, extinção específica por absorção no espectro ultravioleta e potencial antioxidante.

O objetivo desse trabalho foi determinar a composição química e o potencial antioxidante de azeites das espécies Koroneiki, Arbequina e Arbosana, oriundos de oliveiras cultivadas na região oeste de Santa Catarina.

\section{METODOLOGIA}

\subsection{Materiais}

Os azeites avaliados neste trabalho foram obtidos através de moagem e centrifugação a frio de três diferentes variedades, Koroneiki, Arbequina e Arbosana, produzidos nas Unidades Experimentais da Epagri de São Lourenço do Oeste, Chapecó e Caçador, no estado de Santa Catarina.

Todos os reagentes químicos utilizados nas análises possuem grau analítico de pureza.

\subsection{Determinação da composição química}

Determinação da acidez: De acordo com o Instituto Adolfo Lutz (Brasil, 2008), a determinação da acidez pode fornecer um dado importante na avaliação do estado de 
conservação de óleos. Para determinação do índice de acidez utilizou-se o método titulométrico, a partir de solução básica de Hidróxido de sódio 0,1 M. A amostra a ser titulada foi preparado a partir de $2 \mathrm{~g}$ de azeite com $25 \mathrm{~mL}$ de solução éter etílico-álcool na proporção 2:1. Todas as análises foram realizadas em triplicata.

Determinação do índice de peróxido: Este método determina todas as substâncias, em termos de miliequivalentes de peróxido por $1 \mathrm{~kg}$ de amostra, que oxidam o iodeto de potássio nas condições teste. Para essa análise também foi utilizado método titulométrico, segundo instrução do American Oil Chemists Society A.O.C.S. (1992) com análises realizadas em triplicata.

Determinação de compostos fenólicos totais: Para cada amostra de azeite pesaram-se 10 g juntamente com $25 \mathrm{~mL}$ de n-hexano. Essa solução foi então submetida a uma extração líquido-líquido com solução extratora metanol:água na proporção 40:60. Após três extrações, para um volume final de $50 \mathrm{~mL}$, a fase metanol:água foi utilizada para a determinação dos compostos fenólicos. O método de Folin-Ciocalteu baseia-se no princípio de que, em meio alcalino, os compostos fenólicos presentes em uma amostra, reduzem a mistura dos ácidos fosfotúngsticos e fosfomolibdicos, do reagente de Folin, em óxidos de tungstênio e molibdênio de cor azul, tornando possível realizar leituras de absorbância na região do visível do espectro em $765 \mathrm{~nm}$ (Kiralp; Toppare, 2005). A determinação do índice de polifenóis totais foi realizada pelo método de Folin-Ciocalteu descrito por Kiralp e Toppare (2005), com testes realizados em triplicata.

Determinação do índice de iodo pelo método de Wijs: O índice de iodo de um óleo é a medida do seu grau de insaturações e é expresso em termos do número de centigramas de iodo absorvido por grama da amostra (\% iodo absorvido). A determinação do índice de iodo foi realizada pelo método de Wijs, descrito pela metodologia A.O.C.S. (1992), a qual sugere método titulométrico de análise pelo reagente tiossulfato de sódio, sendo que todas as análises foram realizadas em triplicata.

Determinação da capacidade antioxidante total: $\mathrm{O}$ método utilizado foi o descrito por Kumarasamy et al. (2002), com algumas modificações. A amostra foi preparada a fim de obter concentração igual a $500 \mathrm{mg} / \mathrm{mL}$ em solvente clorofórmio, e então diluições foram realizadas para concentrações de 10, 25, 50, 100 e $150 \mathrm{mg} / \mathrm{mL}$. Para cada diluição pipetou-se $100 \mu \mathrm{L}$ e colocou-se em frascos contendo o radical DPPH (2,2-difenil-1-picril-hidrazila), o ensaio realizou-se em triplicata. A leitura das amostras foi realizada por método espectrofotométrico no comprimento de onda de $516 \mathrm{~nm}$.

Determinação do índice de extinção específica: Para a determinação do índice de extinção específica foram dissolvidos $0,25 \mathrm{~g}$ de amostra em $25 \mathrm{~mL}$ de ciclohexano, grau espectrofotométrico. A solução foi adicionada numa cubeta de quartzo, com $1 \mathrm{~cm}$ de caminho óptico e, então determinadas as absorbâncias a $232 \mathrm{~nm}$ e $270 \mathrm{~nm}$, usando o mesmo solvente como referência. 


\section{RESULTADO E DISCUSSÃO}

A Tabela 1 mostra os resultados da determinação do teor de acidez, índice de peróxido e índice de Iodo (Wijs) para as três amostras de azeite analisadas.

Tabela 1 - Valores médios dos parâmetros índice de acidez, índice de peróxidos e índice de iodo dos azeites de oliva extraídos das variedades Arbequina, Koroneiki e Arbosana cultivadas no oeste de Santa Catarina

\begin{tabular}{cccc}
\hline Amostra & $\begin{array}{c}\text { Acidez em ácido } \\
\text { oleico }(\mathrm{g} / 100 \mathrm{~g})\end{array}$ & $\begin{array}{c}\text { Índice de peróxidos } \\
(\mathrm{mEq} / \mathrm{Kg})\end{array}$ & $\begin{array}{c}\text { Índice de iodo } \\
(\mathrm{g} \mathrm{I} / \mathrm{I} / 100 \mathrm{~g})\end{array}$ \\
\hline Koroneiki & $0,719 \pm 0,026$ & $10,46 \pm 0,23$ & $84,08 \pm 4,13$ \\
Arbequina & $0,261 \pm 0,007$ & $16,94 \pm 0,44$ & $76,75 \pm 2,08$ \\
Arbosana & $0,310 \pm 0,008$ & $12,51 \pm 0,70$ & $87,82 \pm 2,04$ \\
Legislação & $1,0^{\mathrm{a}}$ & $20,0^{\mathrm{a}}$ & $75,0-94,0^{\mathrm{b}}$
\end{tabular}
ANVISA.

${ }^{a}$ Valor máximo para azeite de oliva segundo a Resolução RDC no 270, de 22 de setembro de 2005 da ANVISA.

${ }^{\mathrm{b}}$ Valores limites para azeite de oliva segundo a Resolução RDC n ${ }^{\mathrm{o}} 270$, de 22 de setembro de 2005 da

Tecnicamente, o grau de acidez do azeite de oliva é a quantidade de ácidos graxos livres, em relação ao ácido oleico total. Podemos observar na Tabela 1 que todos os azeites avaliados encontram-se abaixo do valor limite de 1,0 g/100g preconizado pela Resolução RCD n ${ }^{\circ}$ 470, de 22 de setembro de 2005 da ANVISA (Brasil, 2005), indicando que os azeites são provenientes de uma matéria-prima de boa qualidade. Um valor alto para o índice de acidez pode ser ocasionado, entre outros fatores, pelo mau tratamento, mau estado de conservação do azeite ou mau estado de conservação dos frutos e tipo de extração (Mello e Pinheiro, 2012). Entre as amostras analisadas, observa-se que o melhor resultado foi encontrado para a amostra proveniente de variedade Arbequina, seguido da Arbosana.

O índice de peróxidos determina a oxidação inicial, a rancificação do azeite de oliva e a deterioração que pode ter havido nos antioxidantes naturais, como tocoferóis e polifenóis. (CARDOSO et. al, 2010). Esse índice, da mesma forma que o teor de acidez, também é utilizado como critério de classificação. Os produtos originados no processo de oxidação, mesmo que em pequenas quantidades, podem alterar o sabor do azeite, inferindo na sua qualidade. Comparando-se os índices de peróxidos das amostras analisadas (Tabela 1) ao limite especificado para a categoria azeite de oliva (Brasil, 2005), que é de $20 \mathrm{mEq} / \mathrm{Kg}$, podese verificar que todas as amostras apresentaram índice inferior ao limite, demonstrando que estes azeites não se encontram em estágio avançado de oxidação, o que contribuiu nas características sensoriais desejadas.

O índice de iodo determina o grau de insaturação de óleos e gorduras através do princípio de que o iodo se adiciona às duplas ligações presentes nas cadeias dos ácidos graxos. Este parâmetro está diretamente relacionado com as propriedades reológicas das amostras, pois as que apresentam baixo índice de iodo, ou seja, as menos insaturadas, são sólidas a temperatura ambiente, e, as que são mais insaturadas, com maior índice de iodo, são líquidas (Mendonça et al., 2008). Segundo o Codex Alimentarius (2003), a determinação do 
índice de iodo cria possibilidades de identificação de adulterações grosseiras, como a mistura de diferentes óleos vegetais. Observa-se que os valores de índice de iodo encontrados para ambos os azeites se encontram dentro da faixa especificada pela legislação (Tabela 2).

Para análise do teor de compostos fenólicos, a curva de calibração obtida a partir de diferentes concentrações de ácido gálico e reagente Folin-Ciocalteu apresentou linearidade na faixa de 0,00092 a $0,016 \mathrm{~g} / \mathrm{L}$, sendo expressa pela equação $y=9,7029 x+0,0027$, a qual representa a relação entre absorbância (y) e concentração (x) em g/L, com um coeficiente de correlação $\left(\mathrm{R}^{2}\right)$ de 0,9967 . Os valores calculados a partir da equação da curva de calibração foram então expressos em termos de $\mathrm{mg}$ por $\mathrm{kg}$ de amostra para melhor interpretação dos resultados (Tabela 2).

Tabela 2 - Valores médios da concentração de compostos fenólicos, potencial antioxidante e extinção específica por absorção na região do ultravioleta $(\mathrm{K})$ dos azeites de oliva extraídos das variedades Arbequina, Koroneiki e Arbosana cultivadas no oeste de Santa Catarina

\begin{tabular}{ccccc}
\hline Amostra & $\begin{array}{c}\text { Compostos } \\
\text { fenólicos }(\mathrm{mg} / \mathrm{kg})\end{array}$ & $\begin{array}{c}\text { Potencial } \\
\text { antioxidante }(\%)\end{array}$ & $\mathrm{K}_{232 \mathrm{~nm}}$ & $\mathrm{~K}_{270 \mathrm{~nm}}$ \\
\hline Koroneiki & $59,40 \pm 0,85$ & $4,86 \pm 0,13$ & 1,973 & 0,175 \\
Arbequina & $102,30 \pm 3,06$ & $18,08 \pm 0,79$ & 2,266 & 0,233 \\
Arbosana & $104,33 \pm 1,53$ & $1,34 \pm 0,08$ & 2,030 & 0,175 \\
Legislação & - & - & $2,600^{\mathrm{a}}$ & $0,25^{\mathrm{a}}$
\end{tabular}
ANVISA.

${ }^{\mathrm{a}}$ Valores limites para azeite de oliva segundo a Resolução RDC n⿳2 270, de 22 de setembro de 2005 da

Os compostos fenólicos são uma importante classe de produtos naturais, responsáveis por processos contra a auto-oxidação e a foto-oxidação nas plantas, pois exercem atividade antioxidante frente aos radicais livres produzidos durante a fotossíntese. Segundo Custódio (2009), estes compostos são encontrados em quantidades significativas nos azeites de oliva quando comparados aos demais óleos vegetais. Por conseguinte, estes compostos são identificados como os principais responsáveis pelas propriedades antioxidantes do azeite de oliva. É importante ressaltar que estes também contribuem nas propriedades sensoriais e coloração dos azeites, participando assim dos atributos positivos avaliados durante a classificação, estando relacionados com a adstringência e o amargor (Morales e Przybylski, 2003; Servili e Montedoro, 2002).

As maiores concentrações de compostos fenólicos foram obtidas nos azeites provenientes das variedades Arbequina e Arbosana, com 102,3 e 104,33 mg/kg, respectivamente, e um valor inferior para a amostra Koroneiki. Estes valores são equivalentes aos valores encontrados por Jorge (2011), o qual relata concentrações na faixa de 81,9 a $184,1 \mathrm{mg} / \mathrm{kg}$ em 20 diferentes azeites de oliva comercializados no Brasil, oriundos de diferentes países, como Espanha, Itália, Grécia, Chile, Argentina e Uruguai. Comparando com azeites oriundos de cultivares do estado do Rio Grande do Sul, Mello e Pinheiro (2012) relatam valores mais elevados, na faixa de 493 a $527 \mathrm{mg} / \mathrm{kg}$. A variedade, o sistema de extração, condições de processamento e armazenamento são alguns dos fatores que 
influenciam o teor final de polifenóis nas amostras de azeites. Segundo Castelo-Branco e Torres (2011), a capacidade antioxidante do azeite de oliva apresenta correlação com o teor de fenólicos totais e individuais, porém, observando os dados da Tabela 2, através da análise do potencial antioxidante pelo método de DPPH não foi possível confirmar essa correlação em todas as amostras analisadas. Segundo alguns autores, ainda não há uma metodologia consolidada referente à análise da capacidade antioxidante de óleos vegetais, e muitas vezes os resultados obtidos são de difícil interpretação. A análise do potencial antioxidante é considerada um desafio analítico devido ao fato de que os métodos disponíveis terem sido desenvolvidos para análise de amostras aquosas, e geralmente adaptações nos ensaios são necessárias para obter melhores resultados (Ninfali, et al., 2001; Castelo-Branco e Torres, 2011).

A extinção específica por absorção na região ultravioleta em $232 \mathrm{~nm}$ e $270 \mathrm{~nm}$ é um importante fator a ser avaliado para a determinação da qualidade do azeite de oliva. $\mathrm{O}$ aumento da absorção em uma dessas regiões, ou em ambas, é indicativo da presença de compostos resultantes de processos oxidativos. Os compostos primários da oxidação apresentam valores máximos de absortividade na faixa entre $220 \mathrm{~nm}$ e $234 \mathrm{~nm}$ (dienos), a partir de $265 \mathrm{~nm}$ são os compostos secundários da oxidação (trienos, aldeídos, cetonas) que apresentam maior absorção (Shahidi e Wanasundara, 2002). Azeites de oliva de boa qualidade e armazenados sob condições adequadas contêm poucos produtos de oxidação e, portanto, valores baixos de absorção a $232 \mathrm{~nm}$ e $270 \mathrm{~nm}$. Quando estes valores superam os limites previstos para cada categoria de azeite, podem indicar a presença de azeites de baixa qualidade ou refinado, óleo de bagaço de oliva ou outros óleos refinados de sementes adicionados ao azeite (Aued-Pimentel et al., 2008). Observa-se na Tabela 2 que todas as amostras avaliadas apresentam valores abaixo do limite previsto na legislação, o que assegura a qualidade dos azeites produzidos na região oeste de Santa Catarina, com destaque para a variedade Arbosana.

\section{CONCLUSÕES}

Os azeites produzidos a partir de oliveiras cultivadas na região oeste de Santa Catarina foram avaliados como de excelente qualidade quanto à sua composição química, pois todos os resultados obtidos estão de acordo com valores previstos na legislação vigente.

\section{REFERÊNCIAS}

AOCS - American Oil Chemists Society. Official methods and recommended practices of the American Oil Chemists' Society. Champaign: AOCS, 1992.

APARICIO, R. Manual del aceite de oliva. In: APARICIO, R.; HARWOOD, J. (Ed.). Autentificación del aceite de oliva. Madri: Mundi-prensa, 2003.

AUED-PIMENTEL, S.; TAKEMOTO, E.; KUMAGAI, E.,E.; CANO, C.,B. Determinação da diferença entre o valor real e o teórico do triglicerídeo ECN 42 para a 
detecção de adulteração em azeites de oliva comercializados no Brasil. Quím. Nova, v. 31, p. 31-34. 2008.

BRASIL. Resolução RDC $n^{\circ} 270$, de 22 de setembro de 2005. Agência Nacional de Vigilância Sanitária (ANVISA), 2005.

BRASIL. Instituto Adolfo Lutz. Métodos físico-químicos para análise de alimentos. $4^{\mathrm{a}}$ ed. $1^{a}$ edição digital. São Paulo: IMESP, 2008.

CASTELO-BRANCO, V. N.; TORRES, A. G. Capacidade antioxidante total de óleos vegetais comestíveis: determinantes químicos e sua relação com a qualidade dos óleos. Rev. Nutr., Campinas, v. 24, n. 1, p. 173-187, 2011.

CARDOSO, G., V.; BARCELOS, M. de F.P.; OLIVEIRA, A. F.; PEREIRA, J. de A.R.; ABREU, W. C.; PIMENTEL. F. de A.; CARDOSO, M. das G.; PEREIRAS, M. C. de A. Características físico-químicas e perfil de ácidos graxos de azeites obtidos de diferentes variedades de oliveiras introduzidas no Sul de Minas Gerais - Brasil. Semina: Ciências Agrárias, v. 13, p. 127-136, jan./mar. 2010.

CODEX ALIMENTARIUS (FAO/WHO). Codex Standard for Olive Oils, and Olive Pomace Oils, CODEX STAN 33 - 1981. Roma, rev. 2. 2003.

COUTINHO, E.F.; RIBEIRO, F.C.; CAPPELLARO, T.H. Cultivo da Oliveira (Olea europea L.), Sistemas de Produção 16, Embrapa. Pelotas-RS, versão on line, 2009.

CUSTÓDIO, T.A.S.; Azeites extra-virgem comerciais: composição em compostos voláteis e relação com parâmetros químicos de qualidade. Universidade do Porto. Portugal. 2009.

JORGE, R.O. Caracterização de azeites virgem extra "Gourmet" varietais e "Blends" comercializados no mercado Rio Grande do Sul. Embrapa Clima Temperado - Teses e Dissertações. Disponível em: http://www.alice.cnptia.embrapa.br/handle/doc/875207. Acesso em: 19 de fevereiro de 2013.

KIRALP, S.; TOPPARE L. Polyphenol content in selected Turkish wines, an alternative method of detection of phenolics. Process Biochemistry, v. 41, p. 236-239, 2006.

KUMARASAMY, Y.; BYRES, M. COX, P.J.; JASPARS., M.; NAHAR, L.; SARKER, S.D. Screening seeds of some Scottish plants for free radical scavenging activity. Phytother. Res., v. 21, p. 615-621, 2007.

MELLO, L.D.; PINHEIRO, M.F. Aspectos de azeites de oliva e de folhas de oliveira. Alim. Nutr., v. 23, p. 537-548, out./dez., 2012.

MENDONÇA, M. A.; BORGO, L. A.; ARAÚJO, W. M. C.; NOVAES, M. R. C. G. Alterações físico-químicas de óleos de soja submetido ao processo de fritura em unidades de produção de refeições no Distrito Federal. Com. Ciên. Saúde, v. 19, p. 115 - 122, 2008. 
MORALES, M.T.; PRZYBYLSKI, R. Oxidación del aceite de oliva. In: Manual del aceite de oliva. Madrid: Mundi-Prensa, p. 442-474, 2003.

NINFALI, P.; ALUIGI, G., BACCHIOCCA, M.; MAGNANI, M. Antioxidant capacity of extra-virgin olive oils, J. American Oil Chemists' Society, v. 78, n. 3, p. 243 247, 2001.

PEIXOTO, A. R. Plantas oleaginosas arbóreas: Oliveira. São Paulo: Nobel, 1973.

PEIXOTO, E.R.M.; Djalva M.N. SANTANA, D.M.N.; ABRANTES, S. Avaliação dos índices de identidade e qualidade do azeite de oliva - proposta para atualização da legislação brasileira. Ciênc. Tecnol. Aliment. v. 18, p. 444-452, 1998.

PERCUSSI, L. Azeite. História, produtores, receitas. São Paulo: Editora Senac, 2006.

PIMENTEL, I. C.; MAGNONI, C. D.; COSTA, R. P. Utilização do azeite de oliva na prevenção e no tratamento de doenças cardiovasculares. Rev. Soc. Cardiol., 17, p. 9-14, jan./mar., 2007.

SHAHIDI, F.; WANASUNDARA, U. N. Methods for Measuring Oxidative Rancidity in Fats and Oils. In: AKOH, C. C.; MIN, D. B. Food Lipids: Chemistry, Nutrition, and Biotechnology. $2^{\mathrm{a}}$ ed., New York: Marcel Dekker, Inc., Cap. 14, 2002.

SERVILI, M.; MONTEDORO, G. Contribution of phenolic compounds to virgin olive oil quality. Eur. J. Lipid Sci. Technol., v. 104, p. 602-613, 2002. 